\title{
A Novel Oxidative Rearrangement of 6-Methoxypyran-2-ones
}

\author{
Serena J. Eade, Robert M. Adlington, Andrew R. Cowley, Magnus W. Walter, ${ }^{\dagger}$ \\ Jack E. Baldwin* \\ Chemistry Research Laboratory, University of Oxford, Mansfield Road, Oxford, OX1 \\ $3 T A$. \\ ${ }^{\dagger}$ Lilly Research Centre, Erl Wood Manor, Windlesham, Surrey, GU20 6PH.
}

\author{
Supporting information
}

General Experimental Methods. Unless otherwise stated, all reactions were carried out under nitrogen. Tetrahydrofuran (THF) was dried by passing through activated alumina columns. Anhydrous $N, N$-dimethylformamide was purchased from Aldrich. All reagents were purchased at the highest commercial quality and used without further purification. NMR spectra were recorded at $400 \mathrm{MHz}$ or $500 \mathrm{MHz}\left({ }^{1} \mathrm{H} \mathrm{NMR}\right)$ and calibrated to the residual solvent peak. The following abbreviations are used for NMR data: s, singlet; d, doublet; sept, septuplet; m, multuplet. Coupling constants are rounded to nearest $0.5 \mathrm{~Hz}$. The following abbreviations are used for IR data: $\mathrm{s}$, strong; m, medium; w, weak; br, broad. Column chromatography was carried out using Sorbsil C60 (40-63mm, 230-240 mesh), silica gel. Reactions were monitored by thin-layer chromatography (TLC) analysis. Spots were visualized by exposure to ultraviolet (UV) light $(254 \mathrm{~mm})$, or by staining with a solution of basic potassium permanganate $\left(\mathrm{KMnO}_{4}\right)$ and then heating. All purified compounds synthesized were determined to be $>95 \%$ pure by ${ }^{1} \mathrm{H}$ NMR. Melting points are uncorrected. The following abbreviations are used for MS data: CI (Chemical ionization), ESI (Electrospray ionization), GCMS (Gas chromatography mass spectrometry), FAB (Fast atom bombardment).

\section{4-hydroxy-6-methoxy-3,5-dimethyl-2H-pyran-2-one (1)}<smiles>COc1oc(=O)c(C)c(O)c1C</smiles> 
${ }^{\mathrm{n}} \mathrm{BuLi}(355 \mathrm{ml}$ of a $2.5 \mathrm{M}$ solution in hexanes, $888 \mathrm{mmol})$ was added to a stirred solution of ${ }^{\mathrm{i}} \mathrm{Pr}_{2} \mathrm{NH}(125 \mathrm{ml}, 869 \mathrm{mmol})$ in dry $\mathrm{THF}(400 \mathrm{ml})$ at $0^{\circ} \mathrm{C}$. After $10 \mathrm{mins}$ a solution of methyl 2-methyl-3-oxopentanoate $(55.69 \mathrm{~g}, 386 \mathrm{mmol})$ in THF $(50 \mathrm{ml})$ was added dropwise. After a further 30 mins the solution was transferred onto $200 \mathrm{~g}$ of solid $\mathrm{CO}_{2}$, and the mixture was agitated for 10 mins. $5 \mathrm{M}$ aq. $\mathrm{HCl}(1 \mathrm{~L})$ was then cautiously added, and agitation continued for another 10mins before the mixture was warmed to RT. The layers were separated, and the aqueous layer was extracted with $\mathrm{Et}_{2} \mathrm{O}(3 \times 600 \mathrm{ml})$ then EtOAc $(1 \times 600 \mathrm{ml})$. The combined organic layers were dried over anhydrous $\mathrm{MgSO}_{4}$, filtered and concentrated under reduced pressure. The crude oily acid was dissolved in DCM (200ml), trifluoroacetic anhydride (150g, 716mmol) was slowly added, and the mixture stirred overnight at RT. The product was concentrated under reduced pressure. The concentrated crude product was split into 10 portions. Addition of $\mathrm{NaOH}(2 \mathrm{M}$, approx. $10 \mathrm{ml})$ at $0^{\circ} \mathrm{C}$ to each portion resulted in precipitation of the product. The resulting mixture was filtered by suction and EtOAc $(10 \mathrm{ml})$ was added to the residue. After $30 \mathrm{mins}$, the recombined product was collected by suction filtration and dried to afford the title compound $(24.37 \mathrm{~g}, 37 \%)$ as a white powder. $\mathrm{R}_{\mathrm{F}} 0.22$ (1:1 30-40 P.E : EtOAc); $\mathrm{mp} 140-146^{\circ} \mathrm{C} ; \mathrm{v}_{\max } / \mathrm{cm}^{-1}(\mathrm{KBr}$ disc) 2928(m, br), 1665(s), 1524(s), 1445(m), 1372(m), 1354(m), 1252(m), 1224(s), $1152(\mathrm{~s}) ; \delta_{\mathrm{H}}(400 \mathrm{MHz}, \mathrm{DMSO}) 1.75\left(3 \mathrm{H}, \mathrm{s}, 5-\mathrm{CH}_{3}\right), 1.79\left(3 \mathrm{H}, \mathrm{s}, 3-\mathrm{CH}_{3}\right), 3.87(3 \mathrm{H}, \mathrm{s}$, 1 '-H), 10.54(1H, broad s, OH); $\delta_{\mathrm{C}}(100.6 \mathrm{MHz}, \mathrm{DMSO}) 7.9\left(3-\mathrm{CH}_{3}\right), 9.9\left(5-\mathrm{CH}_{3}\right)$, 56.5(C-1'), 87.2(C-3), 91.5(C-5), 158.7(C-2), 162.4(C-6), 169.5(C-4). Accurate mass (CI) Calculated for $\mathrm{C}_{8} \mathrm{H}_{11} \mathrm{O}_{4}\left(\mathrm{MH}^{+}\right)$: 171.0657. Found: 171.0655 .

\section{Example of the general procedure for the preparation of $\alpha$-pyrone 2:}

\section{6-methoxy-3,5-dimethyl-2-oxo-2H-pyran-4-yl 4-methylbenzenesulfonate (2a)}

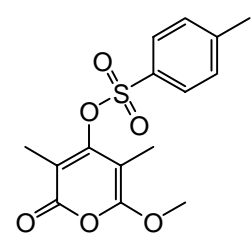

To a stirred solution of pyrone $1(512 \mathrm{mg}, 3.01 \mathrm{mmol})$ in THF:DMF $(5: 1,12 \mathrm{ml})$ at $0^{\circ} \mathrm{C}$ was added $\mathrm{NaH}(240 \mathrm{mg}, 6.02 \mathrm{mmol})$. The suspension was stirred for 5 minutes before the addition of $\mathrm{TsCl}(0.69 \mathrm{~g}, 3.61 \mathrm{mmol})$. The reaction mixture was allowed to warm to RT and stirred for $2 \mathrm{~h}$. The reaction was quenched by addition of sat. aq. $\mathrm{NH}_{4} \mathrm{Cl}(3 \mathrm{ml}) \cdot \mathrm{H}_{2} \mathrm{O}(10 \mathrm{ml})$ was added and the aqueous layer was extracted with 
EtOAc $(2 \times 20 \mathrm{ml})$. The combined organic layers were then extracted with brine $(3 \mathrm{x}$ $40 \mathrm{ml}$ ), dried over anhydrous $\mathrm{MgSO}_{4}$, filtered and concentrated under reduced pressure. The crude product was purified by flash silica gel chromatography (17:3 30-40 P. E. : EtOAc) to yield the title compound (774mg, 79\%) as a white solid. $\mathrm{R}_{\mathrm{F}}$ 0.54 (4:1 30-40 P. E. : EtOAc); $\mathrm{mp}=94-95^{\circ} \mathrm{C} ; \mathrm{v}_{\max } / \mathrm{cm}^{-1}(\mathrm{KBr} \operatorname{disc}) 1728(\mathrm{~s})$, 1637(m), 1596(w), 1544(s), 1385(s), 1336(m), 1243(w), 1200(m), 1180(m), 1079(s); $\delta_{\mathrm{H}}\left(400 \mathrm{MHz}, \mathrm{CDCl}_{3}\right) 1.67(3 \mathrm{H}, \mathrm{s}), 1.78(3 \mathrm{H}, \mathrm{s}), 2.50(3 \mathrm{H}, \mathrm{s}), 4.01(3 \mathrm{H}, \mathrm{s}), 7.38-7.41$ $(2 \mathrm{H}, \mathrm{m}), 7.84-7.88(2 \mathrm{H}, \mathrm{m}) ; \delta_{\mathrm{C}}\left(100.6 \mathrm{MHz}, \mathrm{CDCl}_{3}\right) 8.8,10.8,21.8,56.2,90.3,106.4$, 128.2, 130.2, 133.4, 146.2, 158.6, 161.8, 161.8; Accurate mass (CI) Calculated for $\mathrm{C}_{15} \mathrm{H}_{17} \mathrm{O}_{6} \mathrm{~S}\left(\mathrm{MH}^{+}\right): 325.0746$. Found 325.0940.

\section{6-methoxy-3,5-dimethyl-2-oxo-2H-pyran-4-yl 4-bromobenzenesulfonate (2b)}<smiles>COc1oc(=O)c(C)c(OS(=O)(=O)c2ccc(Br)cc2)c1C</smiles>

The crude product was purified by flash silica gel chromatography (23:2 30-40 P. E. : EtOAc) to yield the title compound (724mg, 63\%) as a white solid. The purified product was recrystallised from $\mathrm{Et}_{2} \mathrm{O}: 30-40$ P.E. under nitrogen. $\mathrm{R}_{\mathrm{F}}$ 0.76 (4:1 30-40 P. E. : EtOAc); $\mathrm{mp}=99-100^{\circ} \mathrm{C} ; \mathrm{v}_{\max } / \mathrm{cm}^{-1}(\mathrm{KBr} \operatorname{disc}) 1736(\mathrm{~s}), 1646(\mathrm{~m}), 1576(\mathrm{~m})$, 1546(m), 1458(w), 1387(s), 1335(m), 1278(w), 1244(w), 1201(m), 1178(m); $\delta_{\mathrm{H}}$ $\left(400 \mathrm{MHz}, \mathrm{CDCl}_{3}\right) 1.72(3 \mathrm{H}, \mathrm{s}), 1.77(3 \mathrm{H}, \mathrm{s}), 4.01(3 \mathrm{H}, \mathrm{s}), 7.75-7.79(2 \mathrm{H}, \mathrm{m}), 7.83-$ 7.88(2H, m); $\delta_{\mathrm{C}}\left(100.6 \mathrm{MHz}, \mathrm{CDCl}_{3}\right)$ 8.3, 10.9, 56.2, 89.9, 106.3, 129.5, 130.3, 133.0, 135.4, 158.7, 161.5, 161.6; Accurate mass (CI) Calculated for $\mathrm{C}_{14} \mathrm{H}_{14} \mathrm{O}_{6} \mathrm{~S}^{79} \mathrm{Br}$ $\left(\mathrm{MH}^{+}\right): 388.9694$. Found 388.9682.

\section{6-methoxy-3,5-dimethyl-2-oxo-2H-pyran-4-yl 4-nitrobenzenesulfonate (2c)}<smiles>COc1oc(=O)c(C)c(OS(=O)(=O)c2ccc([N+](=O)[O-])cc2)c1C</smiles> 
The crude product was purified by flash silica gel chromatography (17:3 30-40 P. E. : EtOAc) to yield the title compound $(614 \mathrm{mg}, 58 \%)$ as a yellow solid. $\mathrm{R}_{\mathrm{F}} 0.48$ (4:1 3040 P. E. : EtOAc); $\mathrm{mp}=131-133^{\circ} \mathrm{C} ; \mathrm{v}_{\max } / \mathrm{cm}^{-1}(\mathrm{KBr} \operatorname{disc}) 1728(\mathrm{~s}), 1637(\mathrm{~m})$, 1608(w), 1543(s), 1451(w), 1406(m), 1389(s), 1342(s), 1314(m), 1245(w), 1201(m), 1187(m), 1100(m), 1085(m); $\delta_{\mathrm{H}}\left(400 \mathrm{MHz}, \mathrm{CDCl}_{3}\right) 1.75(3 \mathrm{H}, \mathrm{s}),, 1.76(3 \mathrm{H}, \mathrm{s})$, 4.02(3H, s), 8.20-8.24(2H, m), 8.46-8.50(2H, m); $\delta_{\mathrm{C}}\left(100.6 \mathrm{MHz}, \mathrm{CDCl}_{3}\right) 8.3,11.0$, 56.3, 89.5, 106.2, 124.7, 129.5, 141.8, 151.2, 158.9, 161.2, 161.3; Accurate mass (CI) Calculated for $\mathrm{C}_{14} \mathrm{H}_{14} \mathrm{NO}_{8} \mathrm{~S}\left(\mathrm{MH}^{+}\right): 356.0440$. Found 356.0446.

\section{4-benzoyl-6-methoxy-3,5-dimethyl-2H-pyran-2-one (2d)}

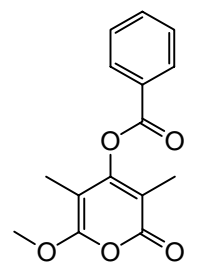

The crude product was purified by flash silica gel chromatography (199:1 DCM : $\left.\mathrm{Et}_{2} \mathrm{O}\right)$ to yield the title compound $(519 \mathrm{mg}, 44 \%)$ as a white solid. $\mathrm{R}_{\mathrm{F}} 0.26(49: 1$ $\left.\mathrm{DCM}: \mathrm{Et}_{2} \mathrm{O}\right) ; \mathrm{mp} 95-97^{\circ} \mathrm{C} ; \mathrm{v}_{\max } / \mathrm{cm}^{-1}(\mathrm{KBr} \operatorname{disc}) 1736(\mathrm{~s}), 1650(\mathrm{~m}), 1566(\mathrm{~m})$, 1451(m), 1380(w), 1340(m), 1259(m), 1241(m), 1197(w), 1176(w), 1112(s), $1048(\mathrm{~m}), 1022(\mathrm{~m}) ; \delta_{\mathrm{H}}\left(400 \mathrm{MHz}, \mathrm{CDCl}_{3}\right) 1.78\left(3 \mathrm{H}, \mathrm{s}, 5-\mathrm{CH}_{3}\right), 1.91(3 \mathrm{H}, \mathrm{s}), 4.03(3 \mathrm{H}$, s), 7.52-7.58(2H, m), 7.67-7.73(1H, m), 8.16-8.21 $(2 \mathrm{H}, \mathrm{m}) ; \delta_{\mathrm{C}}\left(100.6 \mathrm{MHz}, \mathrm{CDCl}_{3}\right)$ 7.4, 9.8, 56.1, 89.5, 104.6, 127.8, 128.9, 130.4, 134.4, 158.6, 161.9, 162.5, 163.9; Accurate mass (ESI) Calculated for $\mathrm{C}_{15} \mathrm{H}_{14} \mathrm{O}_{5}\left(\mathrm{MH}^{+}\right)$: 275.0919. Found 275.0913.

\section{6-methoxy-3,5-dimethyl-2-oxo-2H-pyran-4-yl 2,4,6-triisopropylbenzenesulfonate} (2e)

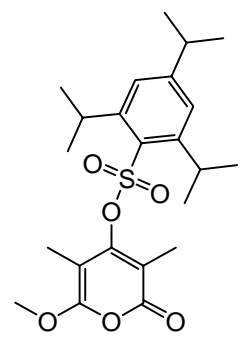

The crude product was purified by flash silica gel chromatography (19:1 30-40 P. E. : EtOAc) to yield the title compound $(924 \mathrm{mg}, 69 \%)$ as a white solid. $\mathrm{R}_{\mathrm{F}} 0.52$ (9:1 3040 P. E. : EtOAc); $\mathrm{mp}=108-109^{\circ} \mathrm{C} ; \mathrm{v}_{\max } / \mathrm{cm}^{-1}(\mathrm{KBr} \operatorname{disc}) 1731(\mathrm{~s}), 1639(\mathrm{~m})$, 
1600(w), 1547(s), 1460(m), 1432(m), 1388(m), 1338(m), 1203(m), 1184(m), 1088(s),

$1035(\mathrm{~m}) ; \delta_{\mathrm{H}}\left(400 \mathrm{MHz}, \mathrm{CDCl}_{3}\right) 1.27(12 \mathrm{H}, \mathrm{d}, \mathrm{J} 7.0 \mathrm{~Hz}), 1.28(6 \mathrm{H}, \mathrm{d}, \mathrm{J} 7.0 \mathrm{~Hz}), 1.71(3 \mathrm{H}$,

s), 1.78(3H, s), 2.95(1H, sept, J 7.0Hz), 3.99(3H, s), 4.07(2H, sept, J 7.0Hz),

7.24(2H, s); $\delta_{\mathrm{C}}\left(100.6 \mathrm{MHz}, \mathrm{CDCl}_{3}\right)$ 8.3, 10.7, 23.5, 24.6, 30.1, 34.3, 56.2, 90.5,

106.4, 124.1, 131.9, 150.1, 154.7, 158.5, 161.8, 162.7; Accurate mass (CI) Calculated for $\mathrm{C}_{23} \mathrm{H}_{33} \mathrm{O}_{6} \mathrm{~S}\left(\mathrm{MH}^{+}\right)$: 437.1998. Found 437.1994.

\section{4,6-dimethoxy-3,5-dimethyl-2H-pyran-2-one (2f)}

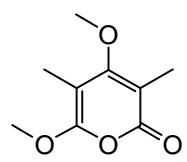

The crude product was purified by flash silica gel chromatography (9:1 30-40 P. E. :

EtOAc) to yield the title compound $(1.07 \mathrm{mg}, 39 \%)$ as a white solid. $\mathrm{R}_{\mathrm{F}} 0.45$ (4:1 30-

40 P. E. : EtOAc); mp 58-60 ${ }^{\circ} ; \mathrm{v}_{\max } / \mathrm{cm}^{-1}$ (KBr disc) 1738(s), 1644(s), 1562(s), 1444(m), 1370(m), 1340(m), $1217(\mathrm{~m}), 1133(\mathrm{~s}) ; \delta_{\mathrm{H}}\left(400 \mathrm{MHz}, \mathrm{CDCl}_{3}\right) 1.84(3 \mathrm{H}, \mathrm{s})$, $1.99(3 \mathrm{H}, \mathrm{s}), 3.81(3 \mathrm{H}, \mathrm{s}), 3.97(3 \mathrm{H}, \mathrm{s}) ; \delta_{\mathrm{C}}\left(100.6 \mathrm{MHz} \mathrm{CDCl}_{3}\right) 7.2,9.8,55.8,60.2$, 89.8, 101.5, 158.7, 163.2, 172.4; Accurate mass (CI) Calculated for $\mathrm{C}_{9} \mathrm{H}_{13} \mathrm{O}_{4}\left(\mathrm{MH}^{+}\right)$: 185.0814. Found 185.0818.

Example of the general procedure for the preparation of $\alpha, \beta$-butenolide 3: methyl 2,4-dimethyl-3-\{[(4-methylphenyl)sulfonyl $]$ oxy $\}-5-0 x 0-2,5-d i h y d r o f u r a n-$ 2-carboxylate (3a)

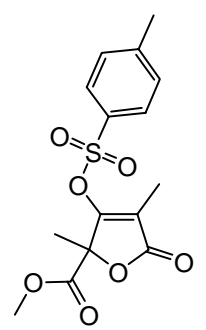

Pyrone 2a $(977 \mathrm{mg}, 2.8 \mathrm{mmol})$ was placed in a 100ml round-bottomed flask which was then flushed with $\mathrm{O}_{2} . \mathrm{CDCl}_{3}(5 \mathrm{ml})$ was added and the flask was left to stand for 2 weeks. The reaction mixture was then concentrated under reduced pressure and the crude material was purified by flash silica gel chromatography (9:1 30-40 P. E. :

EtOAc) to yield the title compound (516mg, 53\%) as a yellow oil. $\mathrm{R}_{\mathrm{F}} 0.75$ (4:1 3040 P. E. : EtOAc); $v_{\max } / \mathrm{cm}^{-1}$ (film) 1748(s), 1694(s), 1596(m), 1494(w), 1446(m), 1385(m), 1309(m), 1261(m), 1196(m), 1180(m), 1125(m), 1089(m); $\delta_{\mathrm{H}}(400 \mathrm{MHz}$, 
$\left.\mathrm{CDCl}_{3}\right) 1.63(3 \mathrm{H}, \mathrm{s}), 1.86(3 \mathrm{H}, \mathrm{s}), 2.50(3 \mathrm{H}, \mathrm{s}), 3.77(3 \mathrm{H}, \mathrm{s}), 7.41-7.45(2 \mathrm{H}, \mathrm{m}), 7.85-$

$7.89(2 \mathrm{H}, \mathrm{m}) ; \delta_{\mathrm{C}}\left(100.6 \mathrm{MHz}, \mathrm{CDCl}_{3}\right) 8.8,19.5,21.8,53.6,82.9,116.1,128.4,130.3$, 132.0, 146.9, 162.1, 167.0, 170.6; Accurate mass (FAB) Calculated for $\mathrm{C}_{15} \mathrm{H}_{17} \mathrm{O}_{7} \mathrm{~S}$ $\left(\mathrm{MH}^{+}\right)$: 341.0695. Found 341.0700.

methyl 2,4-dimethyl-3-\{[(4-bromophenyl)sulfonyl]oxy\}-5-oxo-2,5-dihydrofuran2-carboxylate (3b)

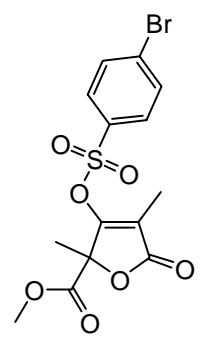

The crude material was purified by flash silica gel chromatography (19:1 30-40 P. E. : EtOAc) to yield the title compound (162mg, 45\%) as a white solid. The purified product was recrystallised from $\mathrm{Et}_{2} \mathrm{O} . \mathrm{R}_{\mathrm{F}} 0.84$ (4:1 30-40 P. E. : EtOAc); mp 105$106^{\circ} \mathrm{C} ; \mathrm{v}_{\max } / \mathrm{cm}^{-1}(\mathrm{KBr} \operatorname{disc}) 1779(\mathrm{~m}), 1731(\mathrm{~s}), 1695(\mathrm{w}), 1575(\mathrm{~m}), 1456(\mathrm{~m})$, 1395(m), 1373(m), 1280(s), 1196(m), 1126(m), 1088(m), 1069(m), 1010(w); $\delta_{\mathrm{H}}$ $\left(400 \mathrm{MHz}, \mathrm{CDCl}_{3}\right) 1.65(3 \mathrm{H}, \mathrm{s}), 1.92(3 \mathrm{H}, \mathrm{s}), 3.78(3 \mathrm{H}, \mathrm{s}), 7.78-7.84(2 \mathrm{H}, \mathrm{m}), 7.84-$ $7.89(2 \mathrm{H}, \mathrm{m}) \delta_{\mathrm{C}}\left(100.6 \mathrm{MHz}, \mathrm{CDCl}_{3}\right) 8.9,19.6,53.6,82.8,116.5,129.8,131.0,133.1$, 134.0, 161.6, 167.0, 170.2; Accurate mass (ESI) Calculated for $\mathrm{C}_{14} \mathrm{H}_{14} \mathrm{O}_{7} \mathrm{~S}^{79} \mathrm{Br}$ $\left(\mathrm{MH}^{+}\right): 404.9644$. Found 404.9659.

methyl 2,4-dimethyl-3-\{[(4-nitrophenyl)sulfonyl $]$ oxy $\}-5-0 x 0-2,5-d i h y d r o f u r a n-2-$ carboxylate $(3 \mathrm{c})$<smiles>COC(=O)C1(C)OC(=O)C(C)=C1OS(=O)(=O)c1ccc([N+](=O)[O-])cc1</smiles>

The crude material was purified by flash silica gel chromatography (17:3 30-40 P. E. : EtOAc) to yield the title compound $(84 \mathrm{mg}, 40 \%)$ as a white solid. $\mathrm{R}_{\mathrm{F}} 0.75$ (4:1 3040 P. E. : EtOAc); $\mathrm{mp} 139-140^{\circ} \mathrm{C} ; \mathrm{v}_{\max } / \mathrm{cm}^{-1}$ (KBr disc) 1784(s), 1755(m), 1699(m), 1611(w), 1542(s), 1455(w), 1379(m), 1307(m), 1273(m), 1194(m), 1138(w), 
$1088(\mathrm{~m}) ; \delta_{\mathrm{H}}\left(400 \mathrm{MHz}, \mathrm{CDCl}_{3}\right) 1.66(3 \mathrm{H}, \mathrm{s}), 1.98(3 \mathrm{H}, \mathrm{s}), 3.80(3 \mathrm{H}, \mathrm{s}), 8.23-8.25(2 \mathrm{H}$, m), 8.25-8.27(2H, m); $\delta_{\mathrm{C}}\left(100.6 \mathrm{MHz}, \mathrm{CDCl}_{3}\right)$ 9.0, 19.8, 53.7, 82.6, 117.1, 124.8, 129.9, 140.5, 151.5, 161.1, 167.1, 169.8; Accurate mass (CI) Calculated for $\mathrm{C}_{14} \mathrm{H}_{17} \mathrm{~N}_{2} \mathrm{O}_{9} \mathrm{~S}\left(\mathrm{MNH}_{4}^{+}\right): 389.0655$. Found 389.0658 .

methyl 3-(benzoyloxy)-2,4-dimethyl-5-oxo-2,5-dihydrofuran-2-carboxylate (3d)<smiles></smiles>

The crude material was purified by flash silica gel chromatography (9:1 30-40 P. E. : $\left.\mathrm{Et}_{2} \mathrm{O}\right)$ to yield the title compound $(181 \mathrm{mg}, 52 \%)$ as a colourless oil. $\mathrm{R}_{\mathrm{F}} 0.36$ (4:1 3040 P. E. : EtOAc); $v_{\max } / \mathrm{cm}^{-1}$ (film) 1779(s), 1698(m), 1600(w), 1452(m), 1387(w), 1235(s), 1147(m), 1067(m), 1036(w), 1012(m); $\delta_{\mathrm{H}}\left(400 \mathrm{MHz}, \mathrm{CDCl}_{3}\right) 1.78(3 \mathrm{H}, \mathrm{s})$, $1.86(3 \mathrm{H}, \mathrm{s}), 3.78(3 \mathrm{H}, \mathrm{s}), 7.52-7.57(2 \mathrm{H}, \mathrm{m}), 7.68-7.73(1 \mathrm{H}, \mathrm{m}), 8.09-8.14(2 \mathrm{H}, \mathrm{m}) ; \delta_{\mathrm{C}}$ $\left(100.6 \mathrm{MHz}, \mathrm{CDCl}_{3}\right)$ 8.6, 20.1, 53.5, 82.8, 114.9, 127.0, 129.0, 130.6, 134.8, 161.1, 163.9, 167.5, 171.0; Accurate mass (GCMS) Calculated for $\mathrm{C}_{15} \mathrm{H}_{15} \mathrm{O}_{6}\left(\mathrm{MH}^{+}\right)$: 291.0869. Found 291.0873.

\section{methyl 2,4-dimethyl-5-oxo-3-\{[2,4,6-triisopropylphenyl)sulfonyl]oxy\}-2,5-} dihydrofuran-2-carboxylate (3e)

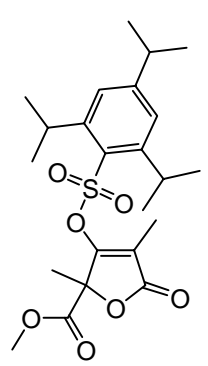

The crude material was purified by flash silica gel chromatography (193:7 30-40 P. E. : $\left.\mathrm{Et}_{2} \mathrm{O}\right)$ to yield the title compound $(181 \mathrm{mg}, 52 \%)$ as a colourless oil. $\mathrm{R}_{\mathrm{F}} 0.81(9: 1$ 30-40 P. E. : EtOAc); $v_{\max } / \mathrm{cm}^{-1}$ (film) 1782(s), 1755(s), 1693(s), 1599(m), 1566(m), 1434(m), 1385(m), 1363(m), 1307(m), 1260(m), 1187(m), 1124(m), 1065(w), $1037(\mathrm{w}) ; \delta_{\mathrm{H}}\left(400 \mathrm{MHz}, \mathrm{CDCl}_{3}\right) 1.27(6 \mathrm{H}, \mathrm{d}, \mathrm{J} 7.0 \mathrm{~Hz}), 1.27(12 \mathrm{H}, \mathrm{d}, \mathrm{J} 7.0 \mathrm{~Hz}), 1.65(3 \mathrm{H}$, s), $1.82(3 \mathrm{H}, \mathrm{s}), 2.95(1 \mathrm{H}$, sept, J 7.0Hz), 3.72(3H, s), 4.02(2H, sept, J 7.0Hz), 7.24(2H, s); $\delta_{\mathrm{C}}\left(100.6 \mathrm{MHz}, \mathrm{CDCl}_{3}\right) 9.0,19.5,23.5,24.5,30.1,34.3,53.4,82.8$, 
$114.8,124.2,130.0,150.8,155.4,162.5,166.9,170.9$; Accurate mass (ESI)

Calculated for $\mathrm{C}_{23} \mathrm{H}_{33} \mathrm{O}_{7} \mathrm{~S}\left(\mathrm{MH}^{+}\right)$: 453.1947. Found 453.1936 .

methyl 3-methoxy-2,4-dimethyl-5-oxo-2,5-dihydrofuran-2-carboxylate (3f)<smiles>COC(=O)C1(C)OC(=O)C(C)=C1OC</smiles>

The crude material was purified by flash silica gel chromatography (4:1 30-40 P. E. : EtOAc) to yield the title compound $(163 \mathrm{mg}, 61 \%)$ as a white solid. The purified product was recrystallised from $\mathrm{Et}_{2} \mathrm{O} . \mathrm{R}_{\mathrm{F}} 0.36$ (4:1 30-40 P. E. : EtOAc); mp 58$61^{\circ} \mathrm{C} ; \mathrm{v}_{\max } / \mathrm{cm}^{-1}(\mathrm{KBr} \operatorname{disc}) 1749(\mathrm{~s}), 1674(\mathrm{~s}), 1455(\mathrm{w}), 1331(\mathrm{~m}), 1267(\mathrm{~m}), 1132(\mathrm{w})$; $\delta_{\mathrm{H}}\left(400 \mathrm{MHz}, \mathrm{CDCl}_{3}\right) 1.66(3 \mathrm{H}, \mathrm{s}), 2.03(3 \mathrm{H}, \mathrm{s}), 3.77(3 \mathrm{H}, \mathrm{s}), 4.14(3 \mathrm{H}, \mathrm{s}) ; \delta_{\mathrm{C}}$ $\left(100.6 \mathrm{MHz}, \mathrm{CDCl}_{3}\right)$ 8.6, 20.2, 53.3, 59.4, 81.8, 97.0, 168.2, 172.0, 175.5; Accurate mass (ESI) Calculated for $\mathrm{C}_{9} \mathrm{H}_{13} \mathrm{O}_{5}\left(\mathrm{MH}^{+}\right): 201.0763$. Found 201.0759.

\section{Methyl 3-hydroxy-2,4-dimethyl-5-oxo-2,5-dihydrofuran-2-carboxylate (3g)}<smiles>COC(=O)C1(C)OC(=O)C(C)=C1O</smiles>

$\mathbf{3 g}$ was separated from $\mathbf{1}$ by addition of EtOAc followed by suction filtration. The filtrate was concentrated under reduced pressure to yield the title compound as a crude yellow oil. $\mathrm{v}_{\max } / \mathrm{cm}^{-1}$ (film) 2999(m, br), 2958(br), 1750(s), 1669(s), 1451(m), 1402(w), 1378(w), 1358(w), 1270(m), 1201(w), 1158(m), 1131(m), 1079(m), 1028(w), 982(w); $\delta_{\mathrm{H}}(400 \mathrm{MHz}, \mathrm{DMSO}) 1.58(3 \mathrm{H}, \mathrm{s}), 1.62(3 \mathrm{H}, \mathrm{s}), 3.70(3 \mathrm{H}, \mathrm{s})$, $12.49\left(1 \mathrm{H}\right.$, broad s); $\delta_{\mathrm{C}}(100.6 \mathrm{MHz}, \mathrm{DMSO}) 7.2,20.7,53.9,82.2,95.6,169.1,173.9$, 174.1; Accurate mass (ESI) Calculated for $\mathrm{C}_{8} \mathrm{H}_{9} \mathrm{O}_{5}\left(\mathrm{M}^{+}\right)$: 185.0454. Found 185.0450.

\section{6-methoxy-3,5-dimethyl-4-oxo-4H-pyran-2-yl 2,4,6-triisopropylbenzenesulfonate} (4e)

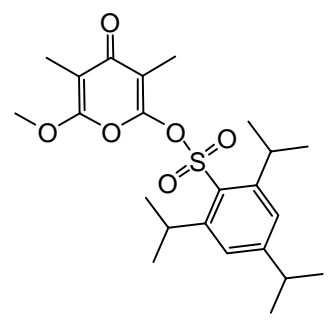


$\mathrm{R}_{\mathrm{F}}$ 0.24 (9:1 30-40 P. E. : EtOAc); mp 119-122 ${ }^{\circ} \mathrm{C} ; \mathrm{v}_{\max } / \mathrm{cm}^{-1}$ (KBr disc) 1687(s), 1611(s), 1463(m), 1429(m), 1383(m), 1322(m), 1247(m), 1156(s), 1127(m), 1074(s), $1036(\mathrm{w}) ; \delta_{\mathrm{H}}\left(400 \mathrm{MHz}, \mathrm{CDCl}_{3}\right) 1.26(12 \mathrm{H}, \mathrm{d}, \mathrm{J} 7.0 \mathrm{~Hz}), 1.27(6 \mathrm{H}, \mathrm{d}, \mathrm{J} 7.0 \mathrm{~Hz}), 1.79(3 \mathrm{H}$, s), 1.90(3H, s), 2.95(1H, sept, J 7.0Hz), 3.36(3H, s), 4.07(2H, sept, J 7.0Hz), 7.26(2H, s); $\delta_{\mathrm{C}}\left(100.6 \mathrm{MHz}, \mathrm{CDCl}_{3}\right)$ 7.0, 8.3, 23.5, 24.6, 30.1, 34.4, 55.1, 99.7, 111.3, 124.2, 130.9, 150.9, 151.1, 155.3, 159.9, 181.7; Accurate mass (CI) Calculated for $\mathrm{C}_{23} \mathrm{H}_{33} \mathrm{O}_{6} \mathrm{~S}\left(\mathrm{MH}^{+}\right)$: 437.1998. Found 437.2004.

\section{2,6-dimethoxy-3,5-dimethyl-4H-pyran-4-one (4f)}

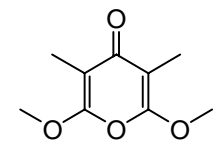

$\mathrm{R}_{\mathrm{F}} 0.17$ (1:1 30-40 P. E. : EtOAc); mp 166-167 $\mathrm{C} ; \mathrm{v}_{\max } / \mathrm{cm}^{-1}$ (KBr disc) 1684(s), 1590(s), 1473(s), 1405(m), 1365(m), 1329(m), 1259(s), 1241(s), 1187(s), 1160(m); $\delta_{\mathrm{H}}\left(400 \mathrm{MHz}, \mathrm{CDCl}_{3}\right) 1.84(6 \mathrm{H}, \mathrm{s}), 3.99(6 \mathrm{H}, \mathrm{s}) ; \delta_{\mathrm{C}}\left(100.6 \mathrm{MHz}, \mathrm{CDCl}_{3}\right) 7.0,56.0$, 99.2, 158.6, 182.5; Accurate mass (ESI) Calculated for $\mathrm{C}_{9} \mathrm{H}_{13} \mathrm{O}_{4}\left(\mathrm{MH}^{+}\right): 185.0814$. Found 185.0814. 